\title{
Aplicación del enfoque sistémico para el posicionamiento estratégico de la gestión del talento humano en la Fuerza Aérea Colombiana (FAC)
}

Application of the systemic approach for the strategic positioning of human resource management in the Colombian Air Force

Citar como: Munar Casas, L. G., Rodríguez Gallego, L. M. y Martínez Lobo, A. P. (2020). Aplicación del enfoque sistémico para el posicionamiento estratégico de la gestión del talento humano en la Fuerza Aérea Colombiana (FAC). CITAS, 6(1). https://doi.org/10.15332/24224529.6364

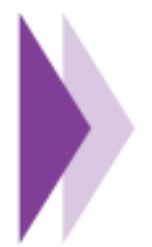

Luz Giovanna Munar Casas ${ }^{1}$; Lina María Rodríguez Gallego ${ }^{2}$; Alicia del Pilar Martínez Lobo ${ }^{3}$

1 Grupo de Investigación en Ciencias Militares Aeronáuticas y Administrativas (GICMA)

2 Grupo de Investigación en Ciencias MilitaresAeronáuticas y Administrativas (GICMA)

3 Grupo de Investigación en Electrónica y Tecnologías para la Defensa Estratégica (TESDA)

Correo electrónico: luz.munar@fac.mil.co lina.rodriguezg@fac.mil.co alicia.martinezlobo@gmail.com 


\section{Resumen}

La Fuerza Aérea Colombiana (FAC) es una institución militar centrada en la jerarquía y en el desarrollo de procesos que están alineados con el orden constitucional, en la que se ha generado un interés significativo por la innovación, la creatividad y la tecnología. De esta manera se plantean estrategias que permiten llevar a cabo una administración del talento humano de excelencia. Por lo tanto, surge la necesidad de fortalecer el sistema de gestión humana, para que propenda por la motivación, el sentido de pertenencia y la fidelización de sus talentos. Teniendo como base la formación de un sistema de gestión humana de excelencia, la Fuerza Aérea Colombiana desarrolla la investigación Modelo estratégico de gestión humana (MEGH)1, bajo un enfoque sistémico fase $I$, que pretende interrelacionar y sincronizar la información de los sistemas informáticos con cada uno de los subsistemas del proceso de gestión del talento humano de manera integral. Esto con el fin de agregar valor a la toma de decisiones de los comandantes, bien sea para la gestión diaria del talento humano en la FAC (traslados, capacitación, evaluación por competencias, planes de desarrollo, evaluación de desempeño, entre otros), como para la toma de decisiones de carácter estratégico (la proyección de perfiles de alto valor, como lo son la selección de cargos críticos o cargos de confianza). Asimismo, se busca obtener información que permita hacer analítica predictiva y prescriptiva, orientada a mejorar los resultados institucionales. El presente artículo reflexivo se enmarca dentro de la primera fase de esta investigación, con la revisión teórica del enfoque sistémico, la gestión del talento humano y su interacción con los macro y microprocesos desarrollados en la FAC.

Palabras clave: enfoque sistémico, fidelización de talentos, gestión humana, modelo de gestión humana.

\section{Introducción}

Desde el 2011, la Fuerza Aérea Colombiana (FAC) desarrolló el sistema de gestión humana por competencias, el cual está orientado a mejorar los procesos institucionales para favorecer la acertada toma de decisiones y potencializar la administración del personal. El modelo que se implementó fue determinado por los siguientes componentes:

$$
\begin{array}{ll}
\text { 1. } & \text { Selección e incorporación } \\
2 . & \text { Desarrollo y formación militar } \\
3 . & \text { Evaluación por competencias }
\end{array}
$$

\section{Abstract}

The Colombian Air Force is a military institution focused on hierarchy and the development of processes that are aligned with the constitutional order, in which significant interest has arisen in innovation, creativity and technology. Thus, strategies are proposed to carry out talent management of excellence. Therefore, there is a need to strengthen the human resource management system to promote motivation, a sense of belonging and loyalty from its talent. Based on the formation of a human resource management system of excellence, the Colombian Air Force is developing the research Strategic model of human resource management (SMHRM)2, under a phase I systemic approach, which aims to interrelate and synchronize in an integral way the information of the computer systems with each of the subsystems of the talent management process. The purpose of this is to add value to the decision\#making processes of commanders, either for the daily management of talent in the Colombian Air Force (transfers, training, competency-based assessment, development plans, performance evaluation, among others), or for strategic decision\#making (projection of high profiles, such as selection processes for critical positions or positions of trust). In addition, it seeks to obtain information that allows for predictive and prescriptive analysis, aimed at improving institutional results. This reflection article is framed in the first phase of this research, with the theoretical review of the systemic approach, talent management and its interaction with macro- and micro-processes developed in the Colombian Air Force.

Keywords: systemic approach, human resource loyalty, human resource management, human resource management model.

$$
\begin{array}{ll}
\text { 4. } & \text { Planes de carrera } \\
5 . & \text { Planes de sucesión } \\
6 . & \text { Compensación. }
\end{array}
$$

La investigación se desarrolla en cuatro fases: la fase I consiste en la caracterización e identificación de los macro y microprocesos que componen el sistema; la fase II se centra en la conceptualización teórica y funcional desde el modelo sistémico, base de la investigación; la fase III corresponde al desarrollo básico de la estructura tecnológica; una vez evaluado y validado el sistema informático, se da inicio a la última fase, la IV, que corresponde a la implementación del sistema en la FAC (figura 1). 
Figura 1. Fases del proyecto "Modelo de gestión estratégica del talento humano de la Fuerza Aérea Colombiana - una visión sistémica (fase 1)". Proyecto "Ingenium 2030"
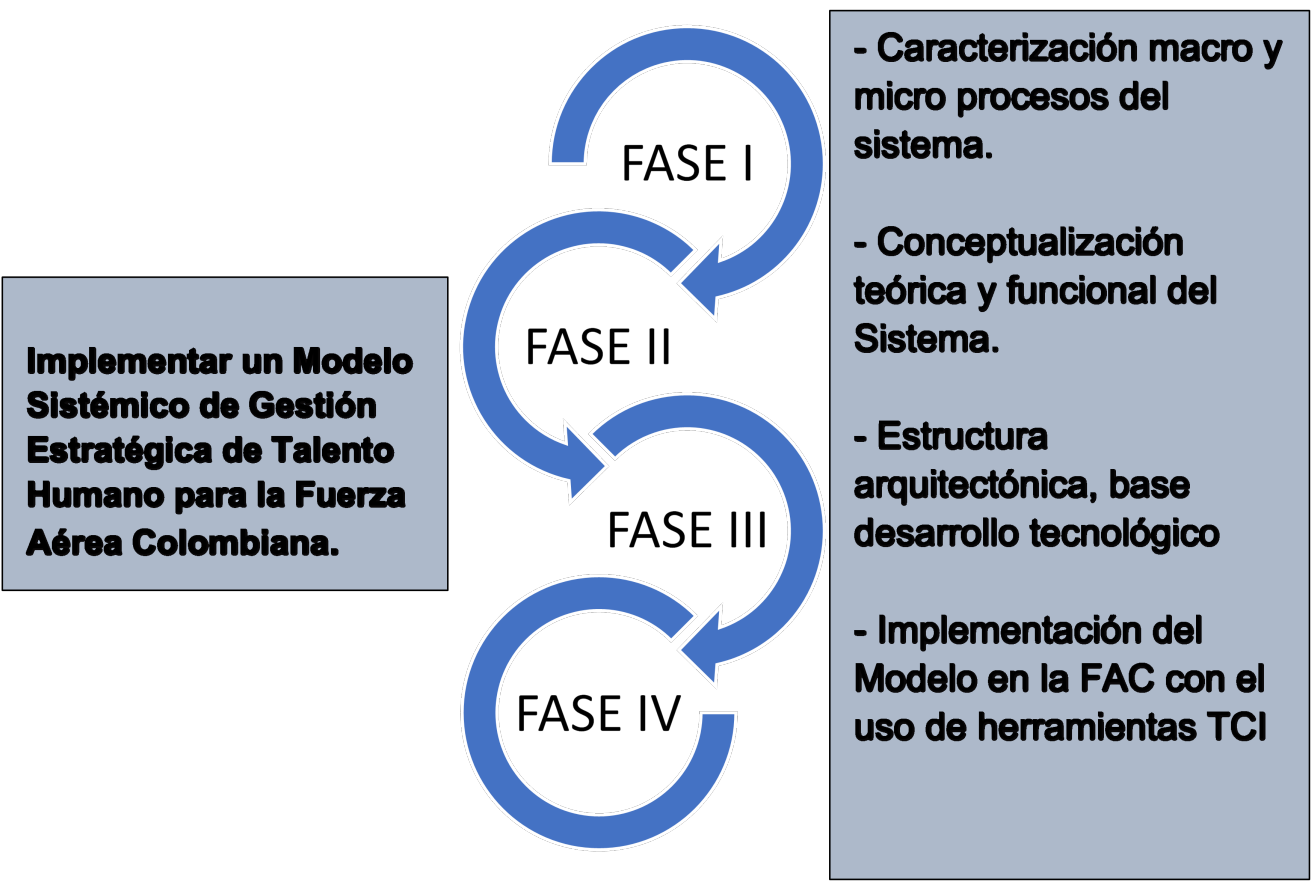

Fuente: elaboración propia.
El artículo se inició con la revisión de la teoría sistémica y sus postulados. Luego se plantearon algunos de los modelos de gestión humana más relevantes y se profundizó en el modelo por competencias implementado por la FAC. Finalmente, se llevó a cabo el análisis del enfoque sistémico y su aporte para la construcción de un modelo de gestión del talento humano para esta institución.

\section{Metodología}

Se realizó una investigación aplicada de desarrollo tecnológico, con un enfoque cualitativo de investigación\#acción con el método inductivo, dado que se basó en la obtención de conclusiones a partir de la observación y análisis de hechos. Esto permitió llegar a interpretaciones válidas, mas no a generalizaciones. El objeto de estudio fueron los macro y microprocesos de la gestión del talento humano utilizados en la FAC.

El diseño de la investigación se estableció en fases secuenciales, a través de las cuales se fueron desarrollando cada uno de los objetivos específicos planteados. La primera fase correspondió al desarrollo del primer objetivo: caracterizar los macro y microprocesos del sistema de gestión humana de la FAC mediante la revisión documental existente y la referenciación con fuentes externas. La segunda fase se centró en el desarrollo de los objetivos 2 y 3 ; y en la cuarta fase se desarrolló la arquitectura general para el sistema tecnológico del modelo estratégico de la gestión del talento humano.

En la figura 2 se observa la secuencia metodológica para el alcance de los objetivos. 


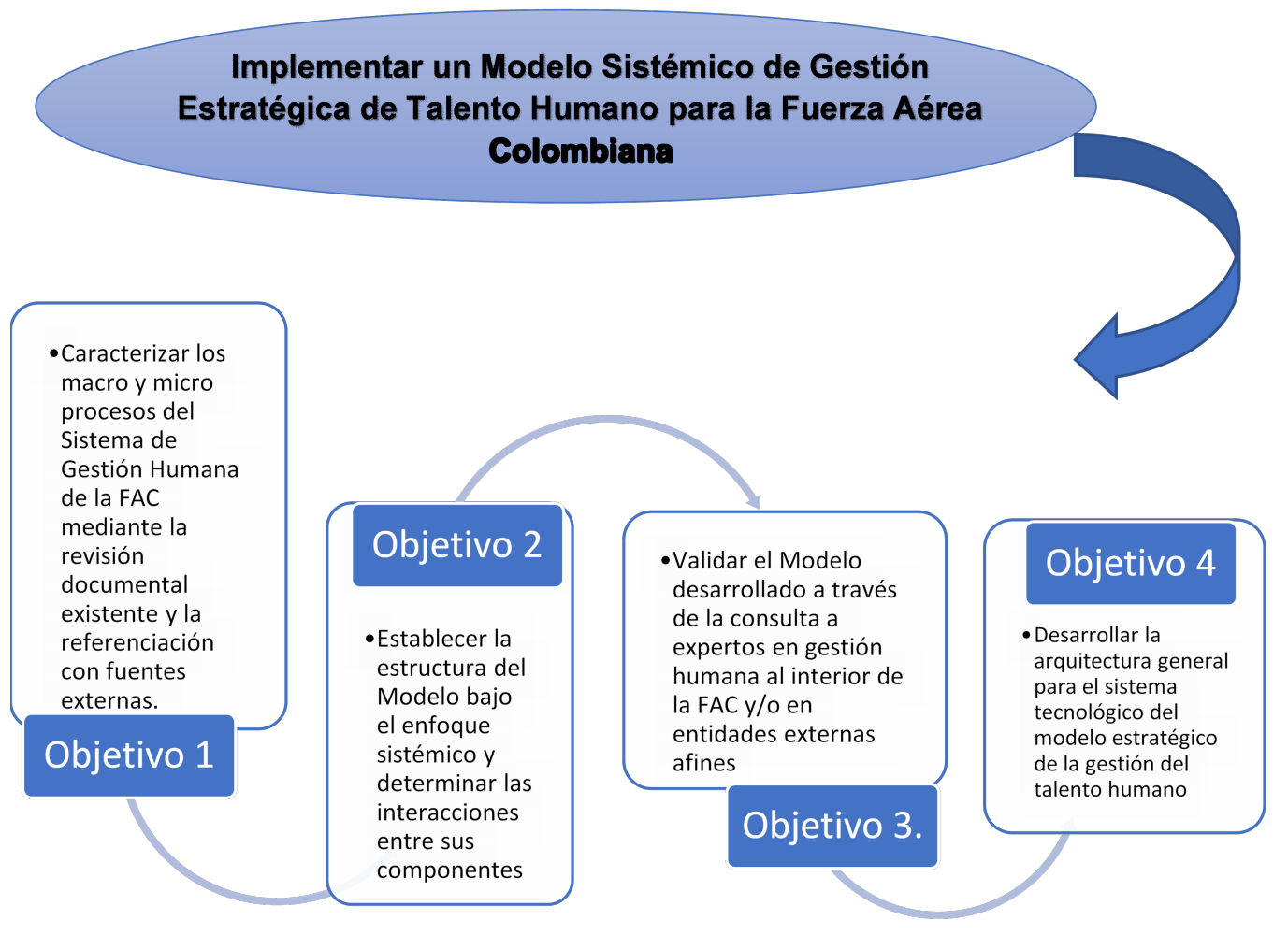

Fuente: elaboración propia.

\section{Marco teórico}

El constructo sistémico es la base teórica que sustenta y argumenta el desarrollo del artículo. Por su parte, los referentes de la teoría sistémica desde la psicología y la teoría de los sistemas de información para el manejo del talento humano a nivel organizacional son el fundamento epistemológico de la investigación.

Para hablar de la teoría sistémica se debe hacer referencia a los conceptos establecidos por Ludwing Von Bertalanffy (1976), quien es considerado el pionero de la teoría general de sistemas. Su enfoque se centra en "la formulación de principios válidos para 'sistemas' en general, sea cual fuere la naturaleza de sus elementos componentes y las relaciones o 'fuerzas' reinantes entre ellos" (p. 37).

Desde un enfoque sistémico, Bertalanffy buscó dar respuesta a la problemática de la psicología en los años 40 del siglo XX: la comunicación en el ser humano y su relación e interacción consigo mismo y su entorno. Este modelo de los sistemas rompe con el paradigma psicológico del momento \#la intervención terapéutica individual\#, dado que reveló una forma holística de observación y análisis al descubrir nuevos fenómenos y estructuras de pensamiento más complejos de ver e interpretar la realidad (Psicólogos en Madrid EU, 2019).

Parafraseando a Bertalanffy, este concepto hace referencia específicamente a que los elementos se organizan y se vinculan en una unidad interrelacionada llamada sistema. Independientemente de los elementos, cada uno depende y afecta al otro, lo cual genera, entonces, una relación causal.

Según la definición dada por Bertalanffy (1987) citado por Trujano (2010), un sistema es:

[...] una serie de elementos interrelacionados con un objetivo común, que se afectan unos a otros, y la característica que los une es la composición que tienen. Es decir, la totalidad, la cual no es sólo la suma de las partes, también la relación entre ellas. Además, cada elemento tiene una función e interactúan entre ellos. Hay diferentes niveles de complejidad de elementos en el sistema (jerarquías diferenciadas) y los elementos se necesitan uno al otro para funcionar. (p. 89)

Por su parte, Salvador Minuchín (1999), citado por Ortiz (2014), también define que un sistema "se trata de un conjunto de 
elementos en interacción que tiene una estructura que determina su funcionamiento, evoluciona con el tiempo y está en interrelación con otros sistemas" (p. 85).

Este nuevo enfoque de la psicología acerca de los sistemas vivos — vista desde el concepto sistémico de Bertalanffy (1976) en relación con los principios rectores de la teoría - afirma que:

Un organismo viviente es ante todo un sistema abierto. Se mantiene en continua incorporación y eliminación de materia, constituyendo y demoliendo componentes sin alcanzar; mientras la vida dure, un estado de equilibrio químico y termodinámico, sino manteniéndose en un estado llamado uniforme (steady) que difiere de aquel. (p. 39)

De igual modo, Ortiz (2014) afirma que "un organismo es dinámico y cuando integra la información, los datos o elementos que consume, puede avanzar, crecer e incluso transformarse" (p. 4).

Lo anterior implica que para poder existir y sobrevivir, un organismo necesita poder intercambiar información entre sus subsistemas \#también denominados componentes\# y, a la vez, con el entorno o suprasistemas. Este concepto está basado en la teoría de los sistemas abiertos, los cuales tienen interacciones externas.

El enfoque sistémico plantea una alternativa de pensamiento, cuyo propósito es ver las totalidades \#desde una mirada holística\#, lo cual conlleva a determinar interacciones entre los elementos, más que en cada uno de ellos por separado. Esto no significa que sea una simple suma de elementos, sino que el pensamiento sistémico posee características propias y diferentes a los elementos por sí solos. Tal como se ha planteado, hace referencia a una concepción holística, que integra sistemas, suprasistemas y subsistemas.

En este orden de ideas, el enfoque sistémico constituye una evolución del pensamiento analítico y lineal a una compresión de la integración entre las partes. Esto es, que migra de una mirada global del objeto estudiado a una comprensión de las interacciones e interdependencias de las partes, entendiendo que la intervención en un solo factor del sistema o en una de sus interacciones va a modificar el comportamiento del sistema total.

La teoría general de los sistemas formulada por Bertalanffy (1976) citado por Arnold y Osorio (1998) plantea diez principios:

1. Totalidad: el sistema trasciende las características individuales de sus miembros, dado que funciona como un todo; es decir, si se altera una parte, afecta al sistema y viceversa, si se afecta el sistema, impacta a las partes (subsistemas).

2. Entropía: los sistemas tienden a conservar su identidad.

3. Sinergia: todo cambio en alguna de las partes afecta a todas las demás y en ocasiones al sistema.

4. Finalidad: los sistemas comparten metas comunes.

5. Equifinalidad: las modificaciones del sistema son independientes de las condiciones iniciales. Cada entidad constituye un sistema abierto que puede alcanzar un "mismo estado final partiendo de diferentes condiciones iniciales y por diferentes caminos" (Bertalanffy, 1976).

6. Equipotencialidad: permite a las partes restantes asumir las funciones de las partes extinguidas.

7. Retroalimentación: los sistemas mantienen un constante intercambio de información.

8. Homeostasis: todo sistema viviente se puede definir por su tendencia a mantenerse estable.
9. Morfogénesis: todo sistema también se define por su tendencia al cambio (Arnold y Osorio, 1998, pp. 1\#12).

Estos principios permiten establecer una organización en la solución de problemáticas, dado que aborda los objetos, situaciones o fenómenos de una manera integral, al identificar y estudiar la interacción que tiene cada una de las partes con el todo. Así mismo, establecen que un conjunto (familia, empresa, grupos social, entre otros) se entiende como un sistema que interactúa internamente con microsistemas \#elementos que lo componen; por ejemplo, en la familia, cada uno de los miembros; en la empresa, cada una de las áreas de trabajo entre otros\#, y que el estado de equilibrio o desequilibrio en cada uno de estos microsistemas afecta positiva o negativamente a los otros sistemas y al sistema en general. De esta forma, el enfoque plantea el análisis de cada una de las partes y del todo en general, y cómo la intervención en una de las partes afecta al sistema total.

Bajo esta orientación, Watzlawick, Beavin y Jackson (1983) definen sistema como el "conjunto de elementos en interacción en donde toda modificación de uno de ellos afecta las relaciones entre los otros elementos" (p. 2). Con base en los conceptos planteados, se puede inferir que el sistema es más que la simple suma de las partes, es la interacción e integración entre los microsistemas que producen un sistema con características y fortalezas diferentes.

Ahora bien, el enfoque teórico de sistemas fue adoptado también por las teorías administrativas al encontrar repuestas sobre el manejo holístico organizacional. Ese punto de vista \#de la organización como una estructura conformada por sistemas, subsistemas y macrosistemas, que pueden ser autónomos, pero a la vez interdependientes\# permite articular e interrelacionar, por un lado, la gestión del personal con las teorías del comportamiento humano $\mathrm{y}$, por el otro, la tecnología informática dentro del sistema cibernético.

Al respecto, Cibanal (2008) citando a Bertatanffy (1976) determina que:

En particular, la teoría de sistemas parece proporcionar un marco teórico unificador tanto para las ciencias naturales como para las sociales, que necesitaban emplear conceptos tales como "organización", "totalidad", "globalidad" e "interacción dinámica", lo lineal es sustituido por lo circular, ninguno de los cuales era fácilmente estudiable por los métodos analíticos de las ciencias puras. Lo individual perdía importancia ante el enfoque interdisciplinario. (p. 2)

Así mismo, en sus postulados, Triviño (1987) considera que:

El enfoque sistémico es un proceso general por el que se analizan y explican elementos correlacionados que constituyen conjuntos sistémicos, permitiendo resolver problemas operativos mediante diversas tecnologías $y$, comprender, cómo se componen y relacionan entre sí las estructuras y los procesos de un programa, de una organización, de un sector e incluso de un sistema de mayor magnitud. (p. 11).

En ese sentido, Triviño (1987) afirma que para "efectos de estructura y función organizacional, la aplicación del enfoque sistémico permite agrupar en forma lógica y ordenada las ciencias, tecnologías, artes y normas utilizadas en el ejercicio de las actividades del sector a través de sus funciones sistémicas de producción”. (p. 11). 
Sobre la importancia de la aplicación de la teoría de los sistemas en las organizaciones, se muestra la manera dinámica requerida para el manejo de procesos propios de la organización en cuanto a personas se refiere. De acuerdo con Triviño (1987):

[...] la identificación de todos sus componentes, variables y mecanismos de dependencia, interrelación y acción recíproca en una organización, permite su análisis en forma integral y la de cada uno de sus macro componentes, facilitando así el estudio de su estructuración y función organizacional. (p. 11)

De esta forma se evidencia la importancia de generar aplicabilidad del enfoque sistémico en la organización, desde una perspectiva centrada en la aceptación de múltiples componentes que crean interacciones, siempre que se den en un orden y con la lógica necesarios para facilitar dichas interacciones.

\section{Teoría de la gestión humana}

Los primeros aportes teóricos de la gestión humana se basan en la administración. Es así cómo se inicia la explicación de la relación entre producción y ser humano, y cobra importancia la concepción teórica de Frederick Winslow Taylor, quien concibe al ser humano en términos de productividad (eficiencia) y hacia un símil entre máquina y hombre. Al respecto, Taylor (1981) indica que:

[...] el propósito más importante de los obreros, debe ser la capacitación y desarrollo de las facultades de cada individuo, de manera tal que puedan efectuar, al ritmo más rápido y con la máxima eficiencia el trabajo que mejor convenga a sus actitudes naturales. (p. 11)

El autor otorga, entonces, mayor importancia a los factores externos influenciados por altos estándares de productividad y eficiencia para el logro de resultados, que a la misma condición humana de los trabajadores. Con ello desconoce los múltiples aspectos que la conforman.

Con el trabajo, el hombre ha logrado satisfacer las necesidades básicas de primer orden, relacionadas con alimento, vivienda y abrigo; sin embargo, en el plano laboral han surgido aspectos del orden comportamental, que corresponden a la motivación y que dan respuesta a la manera determinada de actuar. Esto muestra, como señala McClealland (citado por Alles, 2000) "un interés recurrente para el logro de un objetivo, basado en un incentivo natural; un interés que energiza, orienta y selecciona comportamientos" ( $p$. 42).

$\mathrm{Si}$ las organizaciones promovieran las conductas de sus empleados desde la motivación, desde una perspectiva del orden de los deseos y no de las necesidades, lograrían la identificación de lo que realmente impulsa a las personas a realizar una actividad de determinada manera. Es decir, desde un abordaje emocional y no desde lo instintivo, que al ser satisfecho se extingue sin ninguna trascendencia (Bermúdez y Ortiz, 2011).

Esta visión del hombre en la organización se confrontó con los estudios teóricos de Elton Mayo, Fitz J. Roethlisberger y W. J. Dickson (citados por Sherman y Bohlander, 1994), quienes "con base en los estudios realizados en la Western Electric Companys Hawthorne Works, desempeñaron un papel muy importante en la humanización del lugar de trabajo y desarrollo de formas más humanas de motivación a los trabajadores" (p. 10).

El equilibrio entre la humanización en el lugar de trabajo y la motivación laboral permitió el desarrollo de la administración de los recursos humanos en las organizaciones. Esto dio mayor importancia a la conducta humana y al desarrollo de habilidades para el manejo del personal, que se clasifica, según Sherman y Bohlander (1994), en las siguientes etapas:

1. Reclutamiento y selección

2. Entrenamiento y desarrollo

3. Evaluación del desempeño

4. Administración de las compensaciones y relaciones laborales.

Con el paso del tiempo, las organizaciones han ido evolucionando en el concepto de la perspectiva del hombre en relación con su medio laboral. Esto al enfatizar en la creación e innovación de herramientas que aumenten la productividad, y de igual manera le permitan al individuo un crecimiento en conocimientos, experiencia, calidad de vida, proyección y, en sí, a la identificación y motivación por las actividades que realiza.

En este sentido, Batista y Estupiñán (2018) afirman que:

Tener personas, no significa necesariamente tener talentos, entonces, ¿cuál es la diferencia entre personas y talentos? un talento es un tipo especial de persona y no siempre toda persona es un talento. Para tener talento, la persona debe poseer cualidades diferenciadoras y competitivas que la valoren. Existen cuatro aspectos esenciales en el talento de las personas que reflejan la competencia individual. (Bautista y Estupiñán, 2018, p. 32)

Así, el talento humano es considerado hoy en día como el centro de toda organización, motor principal para la sostenibilidad y el desarrollo de una institución. De esta manera, no constituye un recurso, sino que es el fin en sí mismo; ya que en la medida en que la organización permita su desarrollo y lo valore, motivará un impacto positivo en su productividad.

Batista y Estupiñán (2018) consideran que existen cuatro aspectos importantes que se deben determinar en el personal:

1. Conocimiento: es el saber, es el resultado de aprender a aprender de manera continua.

2. Habilidad: se trata de saber hacer, es la aplicación del conocimiento para resolver problemas y situaciones. Es crear e innovar; en síntesis, es transformar el conocimiento en resultado.

3. Juicio: se trata de saber analizar la situación y el contexto, es tener el análisis crítico, juzgar los hechos, establecer un equilibrio y definir prioridades.

4. Actitud: se trata de saber hacer que ocurran las cosas, actitud positiva y emprendedora. Permite alcanzar y superar metas, asumir riesgos, actuar como agente de cambio, dar valor agregado, llegar a la excelencia y enfocarse a los resultados. Es lo que lleva a la persona a alcanzar la autorrealización (Bautista y Estupiñán, 2018, p. 32).

\section{Modelos estratégicos de gestión del talento humano}

Como punto de partida, es importante conceptualizar qué es gestión estratégica del talento humano. Al respecto, Hendry y Pettigrew (1990) establecen una relación entre el recurso humano y las teorías estratégicas empresariales, y la resaltan como fuente de ventaja competitiva para el individuo. 
De igual manera, autores como Wright y McMahan (1992) coinciden con la relación entre la gestión del talento y la dirección estratégica. Por su parte, Mesch, Perry y Wise (1995), citados por Sanabria colaboradores (2015), la definen como "el proceso de vincular la función del recurso humano con los objetivos estratégicos organizacionales, con el fin último de mejorar el desempeño". (Sanabria et ál., 2015).

Esta definición articula tres conceptos básicos: recurso humano, objetivos estratégicos y mejora del desempeño. De ahí que el talento humano se observa como eje integrador para la ventaja competitiva de una empresa (figura 3 ).

Figura 3. Ejes articuladores para la gestión del talento humano

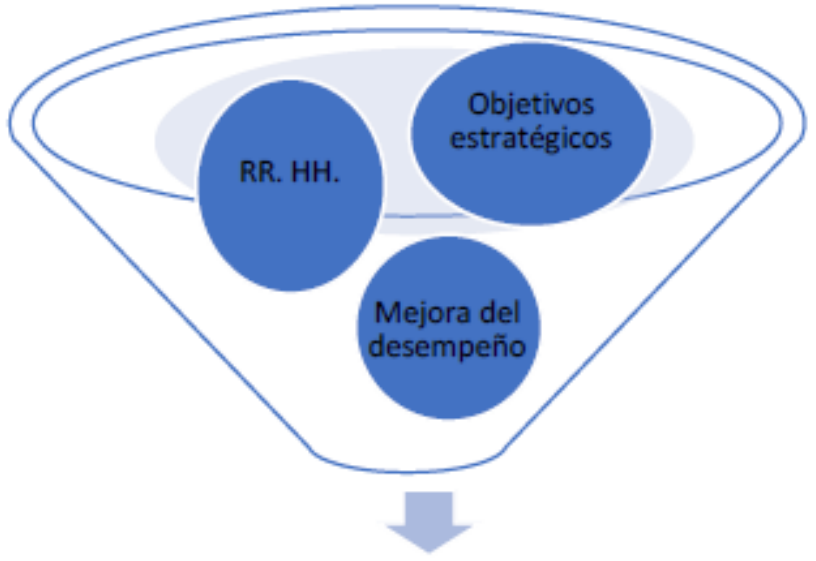

Gestión del talento humano

Fuente: elaboración propia.

Es por ello, que cuando se habla de talento humano en la organización, realmente se hace referencia a personas que cumplan con las competencias técnicas y blandas requeridas para cada cargo y nivel de la organización.

En este orden de ideas, la gestión humana por competencias es entendida de como:

Una herramienta estratégica que tiene como principal objetivo identificar el talento de cada una de las personas que trabajan en una organización y potenciarlo para maximizar sus resultados. Se centra en el impulso de la innovación para el liderazgo transformacional ya que permite a los profesionales conocer su propio perfil de competencias, las áreas de mejora competencial en las que invertir en formación y las competencias requeridas para un puesto. (Larumbe Andueza, 2014, p. 11)

Según estos argumentos, es evidente que las competencias son inherentes al individuo, y la organización debe proveer las estrategias para identificarlas, desarrollarlas y gestionarlas. Esto con el fin de permitir la fidelización de los mejores talentos y garantizar la idoneidad de la persona seleccionada para cada cargo. Lo anterior genera una cadena de valor para la organización, que mejore la toma de decisiones, el trabajo en equipo, la organización del trabajo, la calidad del producto o servicio, y ayude a establecer políticas retributivas justas, que contribuyan a la fidelización de los funcionarios y a aumentar la productividad de la organización.

Por lo tanto, el término gestión del talento humano (GTH) se refiere a los procesos organizacionales que permiten el aprovechamiento de las capacidades, habilidades, destrezas y conocimientos de las personas, para el desarrollo de la organización, de acuerdo con la estrategia de esta.

Para el presente artículo, la gestión del talento humano será entendida como un proceso sistémico enfocado en la estrategia de la organización para el cumplimiento de los objetivos misionales, y potencializando el desarrollo humano, la productividad y la fidelización a la institución.

Lo anterior se logra mediante la implementación de procesos y actividades que actúan como un ciclo en el área organizacional de la gestión humana, y que permite la evolución y crecimiento laboral. De acuerdo con García (2009) la GTH se define como:

[...] el proceso y actividades estratégicas de guía, apoyo y soporte a la dirección de la organización, compuesta por un conjunto de políticas, planes, programas y actividades, con el objeto de obtener, formar, retribuir, desarrollar y motivar el personal requerido para potencializar la organización. (p. 165)

De esta manera, las organizaciones requieren establecer modelos o mecanismos de GTH que permitan dar claridad de los procedimientos, herramientas y condiciones en las que van a evaluar, desarrollar y gestionar a los empleados, de manera que puedan dar cumplimiento a la estrategia de la organización.

Los modelos más recientes de gestión humana son: burocrático, gerencial, de gestión estratégica y orientación a resultados, y de servicio público. Dichos modelos, que se describen a continuación, 
han servido de referentes para las organizaciones en el desarrollo de la gestión del talento humano.

\section{Modelo burocrático}

Su principal precursor fue Max Weber, quien lo planteó como un modelo ideal para brindar una mayor eficiencia a las organizaciones. Petrella (2007), citando a Weber, destaca lo siguiente:

La burocracia concebida por Max Weber se basa en 1) La formalización (tanto de las normas como de los procedimientos) y 2) La centralización (definición de la jerarquía y la autoridad). Ambas dimensiones deben interpretarse desde lo que Weber llama "la racionalidad formal", origen del "orden legítimo" que han de establecer las normas jurídicas. Weber, a través de su conocida trilogía, establece que la legitimidad puede ser "carismática" (se basa en la santidad, heroicidad o en otras cualidades que concurren de forma especial en una determinada persona), "tradicional" (deriva del carácter sagrado que caracteriza a determinadas tradiciones, usos o costumbres) y "racional" (que encuentra su fundamento en el principio de legalidad propio del ordenamiento jurídico). Para Weber, el ejemplo máximo de racionalidad lo constituye la "organización burocrática". (p. 13)

Este modelo aborda la administración del talento humano desde una perspectiva jerárquica, dado que establece niveles de estructura y de autoridad dentro de la organización. Además, diseña normas y reglas que regulan el funcionamiento para establecer directrices, procedimientos y responsabilidades para el cumplimento de los objetivos organizacionales.

\section{Modelo gerencial}

Busca introducir un nuevo conjunto de valores a la gestión humana en el sector público, que incluye la efectividad, la creación de valor público y la orientación hacia el cliente en las organizaciones. Osborne y Gaebler (1992) indican que la mejor manera de alcanzar una mayor efectividad y eficiencia en la gestión de las organizaciones es por medio de la flexibilización de la contratación del personal en la organización y a través de diferentes medidas de seguimiento y evaluación del desempeño, que aseguren una gestión orientada a resultados en todos los niveles organizacionales.

\section{Modelo de gestión estratégica y orientación a resultados}

Este modelo propone la alineación de las prácticas de talento humano con los objetivos misionales de la organización. Además, hace énfasis en un sistema basado tanto en valores públicos como en competencias de los empleados, las cuales son consideradas por su capacidad de crear ventaja competitiva para la entidad (Dennis y Vasu, 2005; Long, 2015).

Este modelo es similar al modelo gerencial, porque enfatiza en los procesos de rotación y de movilidad tanto interna como externa de los funcionarios. Por este motivo se debe impulsar la formación en competencias, para que los empleados exploten todas sus capacidades durante su permanencia en la organización y contribuyan con los objetivos estratégicos de esta (Sanabria, et ál., 2015).

\section{Modelo de servicio público}

En lo relacionado con la gestión del talento humano, Denhardt (2003), referenciado por Sanabria y colaboradores (2015), retoma el valor de las personas sobre la productividad y la necesidad de no confundir emprendimiento con democracia (como un valor clave en lo público). Por otra parte, Sanabria y el equipo también citan a Perry (2010), quien plantea que entre mayor sea la motivación al servicio público de una persona, mayor será la probabilidad de que esta busque pertenecer a una organización pública. Lo mencionado implica que comprobar si efectivamente las organizaciones están conformadas por funcionarios con ciertos atributos puede favorecer el desempeño de esta y el diseño de las acciones relacionadas con la gestión de las personas. (Sanabria et ál., 2015).

\section{Modelo por competencias}

Las competencias, en principio, están asociadas con las habilidades $\mathrm{y}$ aptitudes que son inherentes a una persona y le permiten ser un punto diferenciador con respecto a los demás. Levy (2000) las define como: "comportamientos que unas personas dominan mejor que otras, lo que las convierte en más eficaces en una situación” (p. $35)$.

De ahí, el interés por parte de las empresas en buscar y vincular a personas con atributos que le aportarán \#con el desarrollo de las funciones del cargo\# al éxito y cumplimiento de la misión organizacional. Nace así, la implementación de la gestión del personal mediante un modelo por competencias.

Ruiz (2015) conceptualiza este modelo como un "modelo gerencial mediante el cual se evalúan las competencias específicas para cada puesto de trabajo, y contempla el desarrollo de las competencias adicionales necesarias para el crecimiento personal y profesional de los empleados.

Según lo planteado por los autores, la gestión del talento humano por competencias les permite a las empresas asegurarse que la persona seleccionada para el desarrollo de las actividades requeridas cuente con la idoneidad, preparación y potencial para asumir la responsabilidad del cargo. No obstante, este modelo de gestión va más allá de un proceso de selección e incorporación de personal, pues implica también el desarrollo de los empleados a través de la formación y la oportunidad de crecimiento profesional, económico y social.

La gran ventaja que tiene el uso de este modelo es que utiliza un método de evaluación medible, el cual permite a los gestores o gerentes tomar decisiones con más criterio. De esta manera mejora el clima organizacional, la eficiencia de los empleados y disminuyen los niveles de rotación a los que se enfrenta una organización.

De acuerdo con esta perspectiva, la Fuerza Aérea Colombiana es una organización que concibe a sus funcionarios como un ser humano integral, con valores y principios éticos, que potencia su liderazgo en el campo militar aeronáutico y aeroespacial, comprometido con la misión y visión institucional. Asimismo, está orientando el desarrollo de su personal en el crecimiento del ser humano desde una perspectiva individual, familiar y profesional, para que su actuar sea un referente de ejemplo en la sociedad.

Vidales, Sarmiento y Vargas (2011), en su tesis posgradual, indican que "cuando se ingresa a la Fuerza Aérea Colombiana, la persona hace suyos unos principios y valores que deben responder a un conjunto de códigos culturales y le marcan su devenir histórico, su relación con los demás y consigo mismo" (p. 22).

Desde el 2011, la FAC estructuró el modelo de gestión humana basado en competencias, que "busca desplegar en los líderes militares de la Institución, la habilidad de entender las fortalezas y oportunidades de mejora en personas, procesos y estructura organizacional que permitirán a la Institución posicionarse en el ámbito aeroespacial" (Fuerza Aérea Colombiana, 2011, p. 5). 
Este modelo busca generar una coherencia entre la cultura y la estrategia, vinculando el éxito de la institución con el desarrollo de las personas y reforzando el desempeño superior y la productividad, a través de una cultura de la calidad y del desarrollo del liderazgo personal, misional y estratégico de todos los funcionarios de la institución.

De esta manera, se determinó el modelo con los siguientes componentes, tal como se indica en la figura 4.

Figura 4. Componentes del sistema de gestión humana de la FAC

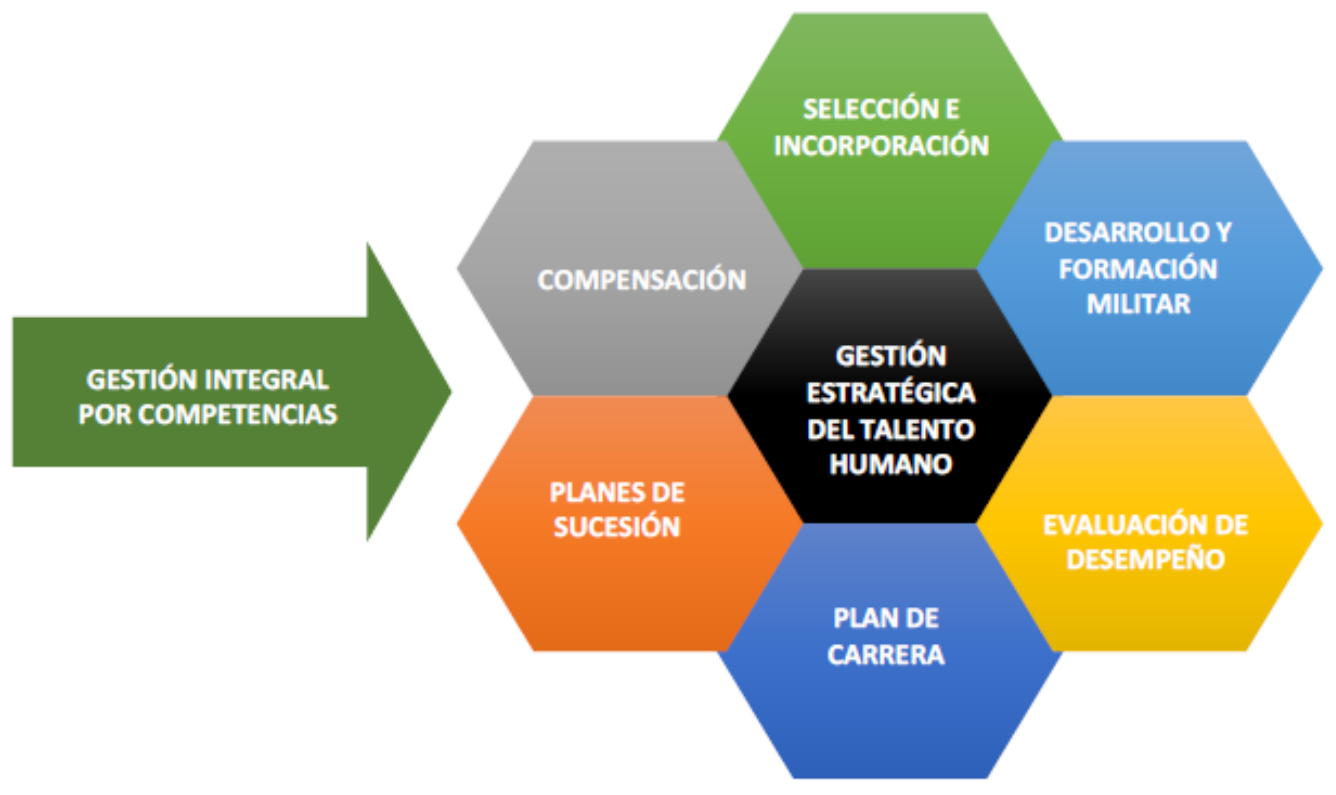

Fuente: tomado de Fuerza Aérea Colombiana (2005).

En el modelo de gestión humana se resalta el componente de evaluación de desempeño, el cual contiene la evaluación por competencias. Este se ha constituido como una herramienta sofisticada para identificar y potencializar el liderazgo militar, enmarcado desde la motivación, las habilidades y las actitudes que requiere el oficial y suboficial de la institución, diferenciando el rol organizacional, nivel profesional y ocupacional de cada funcionario, proyectándolo dentro del plan de carrera.

Durante su implementación, el modelo de gestión humana por competencias de la FAC ha generado óptimos resultados, permitiendo entender la gestión del talento humano en la organización como un potenciador de la estrategia institucional. Sin embargo, pasados diez años de su implementación, y teniendo en cuenta que los procesos institucionales han evolucionado \#que la institución realizó una transformación estructural en el 2018 y que se han identificado diversas oportunidades de mejora\#, se requiere una revisión y ajuste al sistema de gestión humana actual. De esta manera se generó la primera fase de la investigación denominada Modelo de gestión estratégica del talento humano de la Fuerza Aérea Colombiana - una visión sistémica - Proyecto "Ingenium 2030".

El presente escrito corresponde al artículo de resultados de esta primera fase, la cual busca establecer estrategias para que la gestión del talento humano se posicione como un diferenciador estratégico y competitivo, y que la Fuerza Aérea Colombiana se distinga en sus procesos, atrayendo y fidelizando los altos potenciales, quienes tendrán en sus manos la seguridad nacional y el orden constitucional.

Ante la evaluación del sistema de gestión desde el enfoque sistémico, se establece que el sistema de gestión humana es entendido como una herramienta enfocada al cumplimiento de la estrategia de la organización. También se determina que está interconectada con los diferentes componentes que permiten optimizar el talento humano, mejorar el desempeño y la productividad de los empleados, ofrecer un mejor bien o servicio, disminuir los costos y propender por la sostenibilidad de la organización y la fidelización de sus funcionarios.

Con este nuevo modelo, basado en el enfoque sistémico, se busca mejorar la capacidad de relación y comunicación de cada uno de los subsistemas. Es decir, los componentes de selección e incorporación, desarrollo y formación militar, evaluación de desempeño, plan de carrera, planes de sucesión y compensación se interrelacionan con un objetivo común. Dicho de otro modo, aunque cada componente tiene una función específica en el sistema, también debe interactuar con los otros, logrando así esa visión holística trazada por Bertalanffy: las partes afectan al todo; en este caso, al sistema de gestión del talento humano. La figura 5 representa gráficamente el concepto sistémico del modelo. 
Figura 5. Esquema sistémico

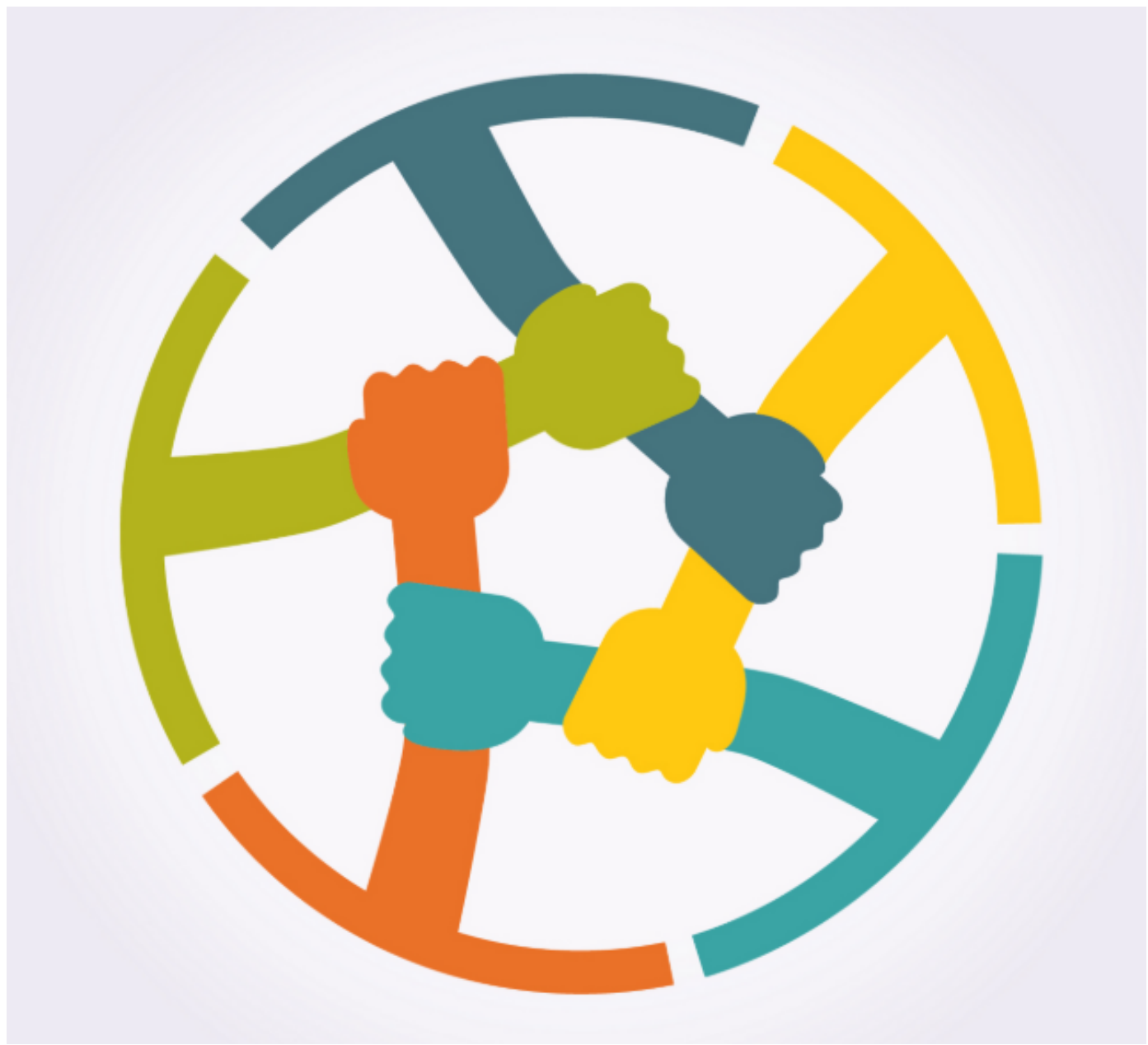

Fuente: tomado de Castro (2018).

En esta investigación el enfoque sistémico es el referente fundamental para el análisis y la descripción del SGH de la FAC como un todo, así como para la identificación de sus piezas. Este parte de los microsistemas proyectados: planeación y presupuestación, incorporación, formación y desarrollo, evaluación y monitoreo del entorno, movilidad y proyección, compensación y retiro. El enfoque sistémico, como se ha esbozado en el desarrollo de este artículo, le permite a la organización establecer la relación e interacción de cada uno de los componentes de los sistemas y subsistemas que lo integran.

De igual manera, este enfoque servirá de base para la intervención, estructuración y construcción de un modelo estratégico de gestión humana (MEGH), que permita repensar la gestión humana dentro de la organización, con un enfoque de pensamiento integrador, interdisciplinario y transdisciplinario, que potencie cada una de las partes del proceso, el macroproceso, las personas y la organización misma.

\section{Conclusiones}

El desarrollo de un modelo de gestión humana sistémico va a generar en la Fuerza Aérea Colombiana un cambio cultural que, alineado con la visión institucional, va a propender por la modernización e innovación de los procesos orientados a potencializar el desarrollo del individuo. De esta manera se genera motivación y fidelización para el cumplimiento de los objetivos misionales de la institución.

El sistema de gestión humana en la Fuerza Aérea Colombiana está fundamentado en una concepción de modelo burocrático, donde se conservan la jerarquización, la autoridad, la subordinación, el cumplimiento de las reglas y los valores. Además, al estar alineado con los cambios tecnológicos, ha impulsado un nivel de flexibilización orientado a potencializar el desarrollo del talento humano, en busca de la fidelización, el nivel de competencias, capacidades y habilidades, la cohesión entre los equipos, la satisfacción laboral y la motivación. Todos estos son diferenciadores que estimulan la identidad militar que representa la organización. 
La armonización de un sistema de gestión humana con una plataforma tecnológica de innovación generará en la institución militar altos estándares de calidad en el manejo del desarrollo del talento humano, con una mayor interacción en cada uno de los procesos, y asegurando así la cualificación del personal.

Partiendo de que la FAC es una organización jerárquica y burocrática, la visión de gestión humana desde su enfoque sistémico pretende desarrollar estrategias para el cumplimiento de los objetivos inherentes a la institución (o institucionales), que promuevan, a su vez, el desarrollo y ascenso profesional de su talento humano.

Las organizaciones hoy en día se enfrentan a un cambio generacional, donde las expectativas del empleador y del empleado son muy diferentes. Por un lado, el empleador busca vincular el mejor talento, con los conocimientos y capacidades que le aporten al desarrollo competitivo de la organización, y que le garanticen productividad y estabilidad. Por otro lado, un empleado busca oportunidades de desarrollo y crecimiento profesional, personal y familiar, desde un sentido de compromiso y pertenencia por la organización.

Dicho cambio imprime un sentido de urgencia hacia cambios que permitan que las organizaciones privadas y públicas encuentren el mejor talento de manera práctica y efectiva, y lo gestionen hacia niveles de alto desempeño.

\section{Referencias}

Alles, M. A. (2000). Dirección Estratégica de Recursos Humanos. Granica.

Arnold, M. y Osorio, F. (1998). Introducción a los Conceptos Básicos de la Teoría General de Sistemas. Cinta de Moebio, 3. https://www.moebio.uchile.cl/03/frprinci.html

Batista, N. y Estupiñán, R. (2018). Gestión empresarial y posmodernidad. Infinite Study.

Bermúdez, H. y Ortiz, O. D. (2011). Entre la necesidad y el deseo. Teuken Bidikay.

Bertalanffy, L. V. (1976). Teoría General de Sistemas. McGraw Hill.

Castro, M. (2018). Teoría sistémica. Medium. https://medium.com/ psicopatolog\%C3\%ADa-del-desarrollo-infantil-2018-2/teor\% C3\%ADa-sist\%C3\%A9mica-773e02784932

Cibanal, L. (2008). Teoría General de Sistemas. http://www.anior te-nic.net/apunt_terap_famil_2.htm

Dennis, M. y Vasu, M. (2005). Supervisory Perceptions of the Impact of Public Sector Personnel Practices on the Achievement of Multiple Goals: Putting the Strategic into Human Resource Management. The American Review of Public Administration. 35(2), 157\#167. https://doi.org/10.1177/0275074004272846

Fuerza Aérea Colombiana [FAC]. (2005, agosto 31). Sistema Gestión Humana FAC. Presentación del Sistema de Gestión Humana por Competneicas FAC, Bogotá D. C. Fuerza Aérea Colombiana.

Fuerza Aérea Colombiana [FAC]. (2011, 18 de julio). Manual para el desarrollo del potencial humano y la capacidad institucional. Capítulo 1. Generalidades. FAC.

García, M. (2009). Los macro\#procesos: un nuevo enfoque en el estudio de la Gestión Humana. Pensamiento \& Gestión. 27, $162 \# 200$.
Hendry, C. y Pettigrew, A. (1990). Gestión de recursos humanos: una agenda para los años noventa. Internacional de Recursos Humanos, $17 \# 42$.

Larumbe Andueza, M. C. (2014). Gestión por competencias [Trabajo fin de Máster, Universidad Pública de Navarra]. http://academica-e.unavarra.es/bitstream/handle/245 4/14006/Carmen\%20Larumbe \%20Andueza.pdf?sequence $=4$

Levy, L. (2000). Gestión de las competencias. Ediciones Gestión. http://cidseci.dgsc.go.cr/datos/Gestion\%20de\%20las \%20competencias-Claude\%20Levy.pdf

Ortiz, D. (2014). Bases para la construcción de una psicología sistémica. Sophia: Colección de Filosofía de la Educación. 16, 83\#98.

Osborne, D. y Gaebler, T. (1992). La reinvención del gobierno. La influencia del espíritu empresarial. Nueva York, NY. 302.

Petrella, C. (2007). Análisis de la teoría burocrática. Revista electrónica de la Facultad de Ciencias Empresariales, 13.

Psicólogos en Madrid EU. (2019, 20 de septiembre). Psicologisenmadrid.eu. https://www.psicologosenmadrid.eu/te oria-general-de-sistemas-de-von-bertalanffy/

Ruiz, L. (2015, 28 de marzo). Modelo de gestión por competencias. Escuela de Organización Industrial. https://www.eoi.es/blogs/m intecon/2015/03/26/modelo-de-gestion-por-competencias/

Sanabria, P., Telch, F., Rodas, A., Astudillo, M. y Estrada, S. (2015, 15 de abril). ¿Cómo Hacer Estratégica la Gestión del Talento Humano en las Organizaciones Públicas Colombianas? Notas de Política.

Sherman, A. y Bohlander, S. W. (1994). Administración de los recursos humanos. Grupo Editorial Iberoamericano.

Taylor, W. (1981). Principios de la administración cientifica y administración Industrial y general. http://jornadassociologia.f ahce.unlp.edu.ar/vii-jornadas-2012/actas/Gismano.pdf

Triviño, J. (1987). El enfoque sistémico: aplicación al subsector de agua potable y alcantarillado (A.P.A.). Ingeniería $e$ Investigación, (16), 10-23. https://doi.org/10.15446/ing.investi g.n16.21574

Trujano, R. S. (2010). Tratamiento Sistémico en problemas familiares. Análisis de caso. Revista electrónica Psicología Iztacala. 13(3), 87\#104. http://www.journals.unam.mx/index.p $\mathrm{hp} / \mathrm{repi} /$ article/view/22593

Vidales, A., Sarmiento, J. E. y Vargas, L. A. (2011). Sistema Educativo para la Fuerza Aérea Colombiana. Universidad de La Sabana.

Watzlawick, P., Beavin, J. H. y Jackson, D. D. (1983). Teoría de la comunicación humana. Interacciones, patologías y paradojas. Editorial Herder.

Wright, P. y McMahan, G. (1992, 1 de junio). Theoretical Perspectives for Strategic Human Resource Management. Journal of Management. 18(2), 295\#320. https://doi.org/10.11 $77 / 014920639201800205$

Notas

1 Proyecto financiado por la Fuerza Aérea Colombiana.

2 Project financed by the Colombian Air Force. 
(c) (1) (3) (2) 